\title{
CogAR: an augmented reality App to improve quality of life of the people with cognitive impairment
}

\author{
Mirko Rossi \\ Department of Computer, Control and \\ Management Engineering \\ Sapienza University of Rome \\ Rome, Italy \\ mirko.rossi@uniroma1.it \\ Mauro Grigioni \\ National Center for Technological \\ Innovation in Public Health \\ Istituto Superiore di Sanità \\ Rome, Italy \\ mauro.grigioni@iss.it
}

\author{
Giuseppe D'Avenio \\ National Center for Technological \\ Innovation in Public Health \\ Istituto Superiore di Sanità \\ Rome, Italy \\ giuseppe.davenio@iss.it
}

\author{
Sandra Morelli \\ National Center for Technological \\ Innovation in Public Health \\ Istituto Superiore di Sanità \\ Rome, Italy \\ sandra.morelli@iss.it
}

\begin{abstract}
The number of people affected by dementia in the world is growing up due to the shift of the population age profile. This paper presents a tool to help and support people who suffer from both cognitive decline age-related and cognitive impairments. Cognitive impairment refers to a person who has trouble remembering, learning, concentrating, or making decisions that affect their daily life. Cognitive impairment varies from mild to severe. With mild impairment, people may begin to notice changes in cognitive functions, while still be able to do their everyday activities; on the other hand, severe levels of impairment can lead to losing the ability to understand the meaning or importance of something in addition to the ability to talk or write, resulting in the inability to live independently. We developed an augmented reality home-made App, CogAR, designed for smart glasses. The App can be a useful tool able to provide more independence to patients during actual daily living activities and in navigating their home, providing cues for memory integration in real time. In this way, elderly people, could stay at home longer without the need to move into a care home environment.
\end{abstract} glasses

Keywords-cognitive impairment, augmented reality, smart

\section{INTRODUCTION}

Cognitive impairment occurs in a continuum, starting with aging-related cognitive decline followed by transition to mild cognitive impairment status, and ultimately to dementia. Mild cognitive impairment does not substantially affect daily function, whereas dementia causes cognitive changes that are potentially severe enough to affect daily function [1] (Fig. 1).

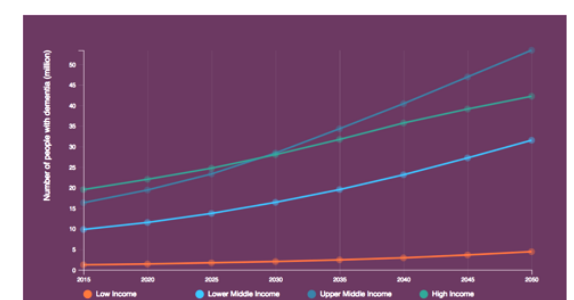

Fig. 2. Evolution of patients' number affected by dementia from 2015 to 2050. The countries are ranked in 4 classes based on their income (low, lower middle, upper middle and high).

As age progresses, in each person a change in his/her brain structure and functions occurs which can provoke a cognitive decline [1]. Cognitive age-related decay involves several symptoms, such as deficits in memory, reduction in level of attention and concentration as well as planning, problem solving and in executive functions [3]. Deficits in executive functions increase the need for help while carrying out activities of daily living (ADL) [4].

All around the world, about 46 million people suffer from dementia. The increase in life expectancy has led to a significant aging of the population and so this number is set to increase to 131.5 million by 2050 [5] (Fig. 2).

People with cognitive impairment are reported as being three times more hospitalized compared to individuals who are hospitalized for other conditions [6]. In many developed countries healthcare cost has overtaken those related to cardiovascular disease and cancer [7].

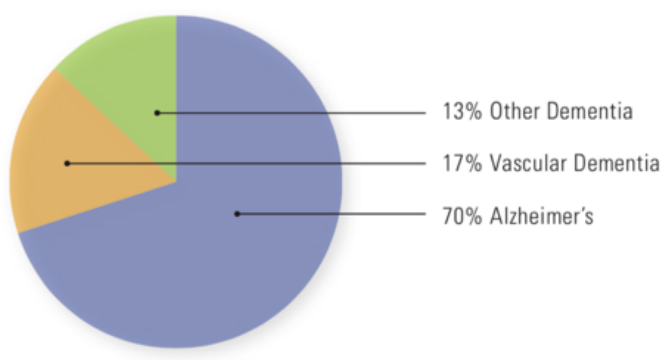

Fig. 3. Causes of Dementia in people aged 71+. [2]
Fig. 1. An individual's progress from healthy cognition to dementia is a continuum. The transition from healthy or normal cognition status to cognitive impairment is not distinct, but blurred. Similar transitions occur between cognitive impairment and dementia. [2] 


\section{A. Causes and signs}

Dementia is characterized by loss or decline in memory and other cognitive abilities [2]. There are over 100 forms of dementia and the most well-known is Alzheimer disease (AD) [8] (Fig. 3). Considering patients with $\mathrm{AD}$, an estimated $4 \%$ are under age $65,6 \%$ are 65 to $74,45 \%$ are 75 to 84 , and $45 \%$ are 85 or older [9]. However, other conditions such as stroke, traumatic brain injury, and developmental disabilities, may cause cognitive impairment [6].

One of the first stages of AD is Mild Cognitive Impairment (MCI), and over time MCI patients are considered at higher risk for dementia [10]. The most common symptom correlated with MCI subjects is the impaired episodic memory; moreover, subjects diagnosed with MCI are associated with a decrease of quality of life and self-esteem, as well as greater social isolation for both categories, patients and their caregivers [4].

A few common signs of cognitive impairment include the following [6], [9]:

- Memory loss that disrupts daily life

- Frequently asking the same questions or repeating the same story over and over.

- Not recognizing relatives or siblings and places.

- Reduced or poor judgment in decision making situation such as how to behave in an emergency.

- Changes in mood or behavior.

- Having issues understanding visual images and spatial orientation.

- Difficulty planning and carrying out tasks, such as following a recipe or keeping track of monthly bills. constantly increased problems with words during speaking or writing.

\section{B. Intervention programs}

The increase of the number of people with cognitive impairment demands to identify a methodology able to detect the decay of cognitive function in an economic and easy way [11]. Studies have focused on the development of efficient treatment strategies.

Elderly people should train their cognitive and motor skill on a daily basis. It is advisable for them to carry out the following activities: physical exercises (e.g., walking), take care of their own house, reading, playing musical instruments, taking part in social events and group activities (e.g., playing cards), learning new skills [12]. In fact, the risk of dementia increases with a lower participation in leisure activities carried out by the person [13]. Previous studies highlighted that consistent physical and social activities help maintaining neuronal plasticity and help increasing brain activity of the elderly people [14], [15], [16], [17], [18], [19].

At this moment, $\mathrm{AD}$ has no cure [8]. Currently, to mitigate the effects of cognitive decline, most common interventions are based on medications' administration [20]. However, it has been also reported the benefit of non-pharmaceutical intervention in elderly [10].

Various non-technological solutions aimed to support elderly people in ADL have been proposed, such as the use of a diary [21], or the modification of the physical environment by removing cabinet doors or employing clear plastic on drawer folders [22].
One of the best ways to maintain the functionality of people in an old age state for the longest possible period is to mitigate the degeneration by stimulating cognitive and physical functions [18]. Cognitive training exercises have been shown to slow down cognitive decay ratio and potentially even reversing it (e.g., [23]), however, the traditional programs of psycho-stimulation have several aspects that make their application difficult, such as high load in human and financial terms [12]. The use of Information and Communication Technologies (ICTs) in this area allows, in principle, to overcome these disadvantages [12]. Hence, as an example of application of ICT tools, researchers considered to evaluate the effectiveness of Serious Games as a training tool for cognitive abilities, obtaining satisfactory results in terms of cognitive and attention skills, reaction times, and a sense of well-being while playing computer games ( [3], [12], [24], [25], [26], [27]). Several studies focused on the use of Virtual Reality (VR) ([3], [12], [25], [28], [29], [30]). Serious Games and VR experiences allow users to face situations similar to those encountered in real life, but with complete safety, control and low-cost environment [3]. In this way, therapists can assess patients' performance.

The artificial nature of laboratories enhances the internal validity but reduces the ecological validity aspect. Traditional measures in lab, being out of the context of real life, could limit the elderly performance and improvement by avoiding the benefit of the experience and the confidence gain expected by a familiar context [31]. The advancement of technology results in the possibility of supporting the people with cognitive impairment in their own house [4]. By bringing technology in each user's daily life, this changes the type of the assessment: from short and episodic monitoring to continuous and real time evaluation. Several technologies have been studied with the purpose of supporting people during ADL. These ranges from single simple prompts [32], [33], [34] to more complex input sequences [35], [36]. A solution is offered by Smart Homes, that allows to control patient's health status and detect specific events, such as falling [11], [37], [38], [39], [40]. Smart Homes can offer an evaluable environment, efficient from an ecological point of view [39]. The disadvantage is the demand of many sensors, which is generally associated with a high financial expense.

A cheaper solution is the use of wearable sensors such as Head Mounted Displays (HMDs) for augmented reality. HMDs are rapidly evolving. In the last decade there have been great improvements both from the point of view of design and portability, and from the point of view of technology in terms of display-type (from Poly-silicon TFT active matrix to Mono Crystalline Silicon Active Matrix), pixel number (from 518,400 to 921,600 pixels) and refresh rate (from 60 to $30 \mathrm{~Hz}$ ).

The HMD devices could be an efficient tool to hinder the cognitive decay of elderly people. Augmented reality (AR) is a technology where a user's view or vision of the real world is enhanced or augmented with additional information generated from a computer model. The AR interface can decrease the cognitive load [32], [33], [34], [36], [41], [42]. In fact, elderly people are prone to use mental representations to enhance short-term memory [34]. In comparison to $\mathrm{VR}, \mathrm{AR}$ is considered more user friendly, since it does not isolate the user from his/her environment. Furthermore, AR technology allows to combine both cognitive with physical exercises. 


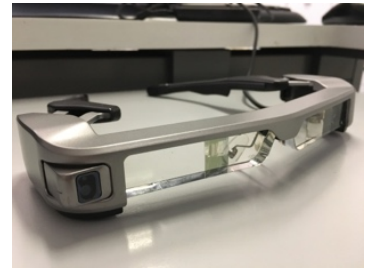

Fig. 4. The commercial HMD used in this study.

For these reasons, we have decided to develop an AR marker-based Application, $\operatorname{Cog} A R$, to assist patients in their ADL - at home and also away from home. The AR system aims to show (symbolically and/or textually) the hidden spots, such as rooms behind doors or objects in the cupboards, and to warn that a contact with an object may be dangerous, such as electrical sockets, stoves, ovens or other house utilities [43].

This proposed tool mainly targets subjects who are living alone: about one-third of all people with dementia are living on their own [44]. They are considered a particularly vulnerable group, at an increased risk for unmet social, environmental, psychological and medical attention.

\section{MATERIALS AND METHODS}

Android is a mobile operating system designed primarily for smart devices. CogAr is an Android marker-based augmented reality App developed for a commercial HMD.

\section{A. Set-up}

To be run, the App requires only the HMD device and a printed image (marker) which we have associated with augmented information (Augmented Object, AO) via software. The HMD is equipped with a series of integrated sensors, including a camera embedded on its right side (Fig.4). In order to program the markers that can be recognized by the HMD and to associate each of them with an AO, we used Unity $3 \mathrm{~d}$, a cross-platform game engine, and a plugin provided by the HMD's manufacturer. The plugin allows you to choose and upload specific 2-D images or 3-D models that will act as markers. Through Unity it is possible to associate each of these markers with an AO, positioned close to the marker, which can be represented by a customizable image, text or multimedia content (audio or video).

\section{B. User interface}

When the user wears the HMD, he experiences the surrounding real-world environment while the software is generating visual elements that are overlaid on the real-life visualization [8]. We set specific markers on the physical objects of interest, as suggested by the National Institute on Aging (USA) [43]. Each of these markers is associated to a particular AO (text, picture, ...), which can be programmed based on the subject's preferences and needs. When a marker falls within the camera's field of view, the system shows the $\mathrm{AO}$ to the user, overlaid on the marker position.

Furthermore, we decided to show on top of the screen of the HMD the fundamental user contacts (i.e.: police, ambulance and caregiver telephone number). Thus, if the user needs it, he/she can easily reach the emergency contact (e.g., police, ambulance or caregiver) handy.

Finally, thanks to the camera being always active while the application is running, and to a free of charge mirroring App available in PlayStore ("WiFi-Display(miracast)"), which allows to share the user's field of view, the caregiver has the possibility to check at any time the patient's activity from his/her smartphone and therefore the ability to quickly identify potentially dangerous situations.

Fig. 5 and Fig. 6 explain two examples of the CogAR. The top panel of each figure shows the users' field of view when he/she puts on the HMD and the marker is out of the camera's field of view; on the bottom panel, the camera identifies the marker and the associated augmented objects are shown.
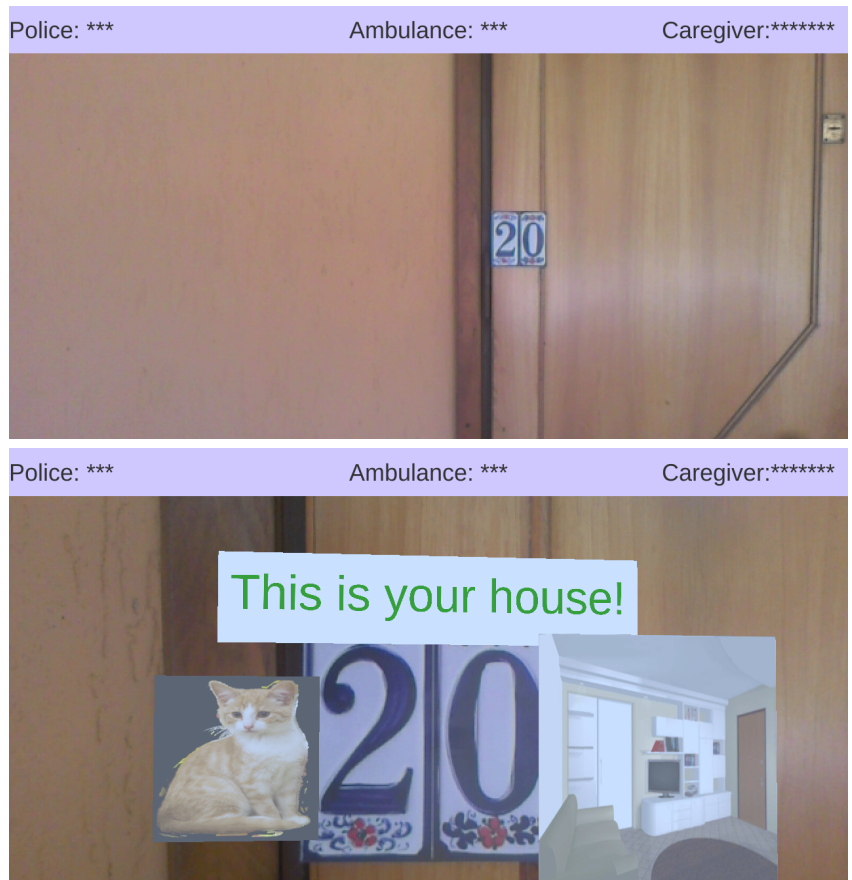

Fig. 5. The marker is applied on the entry door of the subject's house (top); when the user looks at the marker, through the HMD, information that recalls his/her own home are shown (bottom)

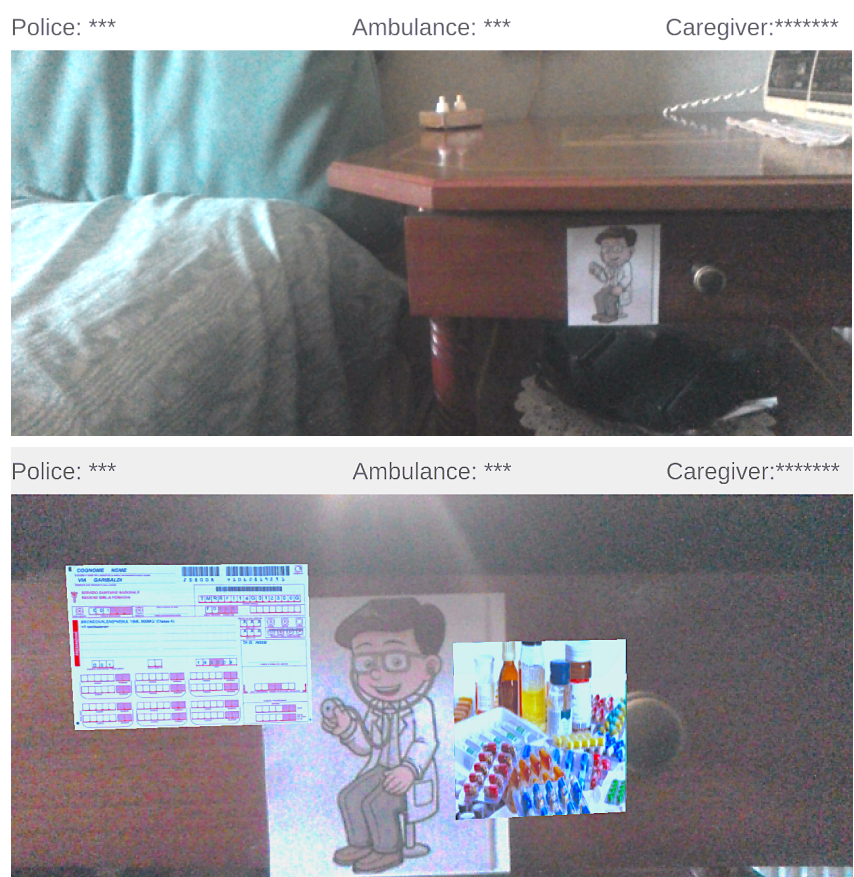

Fig. 6. The marker is applied on the bedside table where the user keeps the medicines (top); the view through the HMD when the user looks at the marker that shows the contents of the drawer (bottom) 


\section{CONCLUSION AND FUTURE WORK}

The AR-supported home environment can be useful for patients while navigating the home itself, providing cues for

memory integration. This patient empowerment should lead to an improved confidence during ADL and, ultimately, a higher degree of physical activity. The possibility provided by the AR to merge cognitive exercises with physical activity is expected to make the support intervention more effective. In fact, increasing level of physical activity has now been identified with certainty as a protective/preventive factor against the progression of neurodegenerative diseases [14], [19].

Elderly people may find difficult accessing and using technology as well as facing deficits in learning new information. This would limit the ability of persons with dementia to acquire the conceptual knowledge needed to interact with a novel interface. Furthermore, impaired attentional control would also disrupt their ability to adopt unfamiliar procedures.

The tool presented in this paper could enhance the quality of life of elderly people breaking down these barriers for the following reasons:

- $\quad$ No need to learn new skill

- Any input device or button to be pressed is not required

- It is possible to know where any object is located, without the need to open all the doors or all the drawers, un-necessary movements are limited and so the environment becomes safer.

Therefore, we are confident that our system could meet the criteria of compliance (always having the device by hand), dignity and usability. With the purpose to assess the latter, we plan to carry out an experimental study, initially involving elderly healthy people.

\section{ACKNOWLEDGMENT}

This study was supported by the project "High-end and Low-End Virtual Reality Systems for the Rehabilitation of Frailty in the Elderly" (Project Code: PE-2013-02355948), funded by Italian Ministry of Health.

\section{REFERENCES}

[1] Canadian Task Force on Preventive Health Care, "Recommendations on screening for cognitive impairment in older adults," Canadian Medical Association Journal, 2016; 188:37-46.

[2] Alzheimer's Association, “Alzheimer's disease facts and figures," Alz Dem J Alz Assoc 5(3):234-70, May 2009.

[3] M. J. Rodriguez-Fortiz, J. Revelles-Moreno, S. Rute-Perez, C. Rodriguez-Dominguez, M. L. Rodriguez-Almendros, P. CanoOlivares, M. V. Hurtado-Torres, "Serious games for the cognitive stimulation of Elderly People," $4{ }^{\text {th }}$ International Conference on Serious Games and Applications for Health (SeGAH), Orlando, Florida, 2016.

[4] J. P. Wherton and A. F. Monk, "Technological opportunities for supporting people with dementia who are living at home," International Journal of Human-Computer Studies 66(8), 571-586, 2008.

[5] J. Hayhurst, "How Augmented Reality and Virtual Reality is Being Used to Support People Living with Dementia—Design Challenges and Future Directions" in Augmented Reality and Virtual Reality, Springer, pp. 295-305, 2018.

[6] Centers for Disease Control and Prevention, "Cognitive impairment: A call for action now!," U.S. Department of Health and Human Services, February 2011

[7] M. Valenzuela and P. Sachdev, "Can Cognitive Exercise Prevent Onset of Dementia? Systematic Review of Randomized Clinical Trials with
Longitudinal Follow-up," American Journal of Geriatric Psychiatry, 17(3), pp. 179-187, 2009.

[8] B. Aruanno, F. Garzotto, and M. C. Rodriguez, "HoloLens-based Mixed Reality Experiences for Subjects with Alzheimer's Disease," In Proceedings of the $12^{\text {th }}$ Biannual Conference on Italian SIGCHI Chapter (CHItaly '17), Article 15, 9 pages, September 2017.

[9] Alzheimer's Association, “Alzheimer's disease facts and figures," Alz Dem J Alz Assoc 7(2):208-244, March 2011.

[10] J. Zhao J, H. Li, R. Lin, Y. Wei, and A. Yang, "Effects of creative expression therapy for older adults with mild cognitive impairment at risk of Alzheimer's disease: a randomized controlled clinical trial," Clin Interv Aging. 13:1313-1320, July 2018.

[11] A. Konig, C. F. Crispim Junior, A. Derreumaux, G. Bensadoun, P. D. Petit, F. Bremond, R. David, F. Verhey, P. Aalten, and P. Robert, "Validation of an automatic video monitoring system for the detection of instrumental activities of daily living in dementia patients," Journal of Alzheimer's Disease, 44(2), 675-685, 2015

[12] A. López-Martínez, S. Santiago-Ramajo, A. Caracuel-Romero, C. Valls-Serrano, M. J. Hornos, and M. J. Rodríguez-Fórtiz, "Game of gifts purchase: Computer-based training of executive functions for the elderly", Proceeding of IEEE $1^{\text {st }}$ International Conference on Serious Games and Applications for Health (SeGAH 2011), pp. 1-8, 2011.

[13] J. Verghese, R. B. Lipton, M. J. Katz, C. B. Hall, C. A. Derby, G. Kuslansky, A. F. Ambrose, M. Sliwinski, and H. Buschke, "Leisure activities and the risk of dementia in the elderly," $\mathrm{N}$ Engl $\mathrm{J}$ Med, 348:2508-2516, June 2013.

[14] A. Verdelho, S. Madureira, J. M. Ferro, H. Baezner, C. Blahak, A. Poggesi, M. Hennerici, L. Pantoni, F. Fazekas, P. Scheltens, G. Waldemar, A. Wallin, T. Erkinjuntti and D. Inzitari,"Physical activity prevents progression for cognitive impairment and vascular dementia: results from the LADIS (Leukoaraiosis and Disability) study," Stroke, 43(12): 3331-5, December 2012

[15] K. Hauer, P. Ullrich, I. Dutzi, R. Beurskens, S. Kern, and M. Schwenk, "Effects of standardized home training in patients with cognitive impairment following geriatric rehabilitation: a randomized controlled pilot study," Gerontology, 63(6):495-506, 2017.

[16] M. Hagovska and I. Nagyova, "The transfer of skills from cognitive and physical training to activities of daily living: a randomised controlled study," Eur J Ageing, 14(2):133-142, September 2016.

[17] H. Shimada, K. Ishii, H. Makizako, K. Ishiwata, K. Oda, and M. Suzukawa, "Effects of exercise on brain activity during walking in older adults: a randomized controlled trial," J Neuroeng Rehabil, 14(1):50, May 2017.

[18] Y. Lin, H. Mao, Y. Tsai, and J. Chou, "Developing a serious game for the elderly to do physical and cognitive hybrid activities", IEEE $6^{\text {th }}$ International Conference on Serious Games and Applications for Health(SeGAH), pp. 1-8, 2018.

[19] X. Kou, D. Chen, and N. Chen, "Physical Activity Alleviates Cognitive Dysfunction of Alzheimer's Disease through Regulating the mTOR Signaling Pathway," Int. J. Mol. Sci, 20(7):1591, April 2019.

[20] NHS, "What are the treatments for dementia?," NHS, 17 June 2017. Available:http://www.nhs.uk/conditions/dementiaguide/pages/dementia-treatment.aspx

[21] I. G. Hanley and K. Lusty, "Case histories and shorter communications: memory aids in reality orientation: a single-case study," Behaviour Research and Therapy 22(6), 709-712, 1984.

[22] R. L. Beard, J. Knauss, and D. Moyer, "Managing disability and enjoying life: how we reframe dementia through personal narratives," J. Aging Stud 23(4):227-235, December 2009.

[23] K. Ball, D. B. Berch, K. F. Helmers, J.B. Jobe, M. D. Leveck, M. Marsiske, J. N. Morris, G. W. Rebok, D. M. Smith, S. L. Tennstedt, F. W. Unverzagt, and S.L. Willis, "Effects of cognitive training interventions with older adults: a randomized clinical trial," JAMA, 288 (2002), pp. 2271-2281, November 2002.

[24] H. Chi, E. Agama, and Z. Prodanoff, "Developing serious games to promote cognitive abilities for the elderly," 2017 IEEE 5th International Conference on Serious Games and Applications for Health (SeGAH), pp. 1-8, 2017.

[25] J. W. Burke, M. D. McNeill, D. Charles, P. Morrow, J. H. Crosbie, and S. M. McDonough, "Optimising engagement for stroke rehabilitation using serious games," Visual Computer, vol.25, pp. 1085-1099, 2009.

[26] I. Di Loreto, and A. Gouaich, "Mixed reality serious games: The therapist perspective," Proceeding of IEEE 1st International 
Conference on Serious Games and Applications for Health (SeGAH 2011), pp. 1-10, 2011.

[27] A. Conconi, T. Ganchev, O. Kocsis, G. Papadopoulos, F. FernándezAranda, and S. Jiménez-Murcia, "PlayMancer: A serious gaming 3D environment", Int. Conf. on Automated Solutions for Cross Media Content and Multi-channel Distribution (AXMEDIS`08), IEEE Press, pp. 111-117, 2008.

[28] S. Serino S, E. Pedroli, C. Tuena, G. De Leo, M. Stramba-Badiale, K. Goulene, N. G. Mariotti, and G. Riva, "A novel virtual reality-based training protocol for the enhancement of the mental frame syncing in individuals with Alzheimer's disease: a development-of-concept trial," Front Aging Neurosci 9:240, July 2017.

[29] A. Rizzo and G. J. Kim, "A swot analysis of the field of virtual reality rehabilitation and therapy," Presence: Teleoperators and Virtual Environment 14 (2), pp.119-146, 2005.

[30] V. Manera, E. Chapoulie, J. Bourgeois, R. Guerchouche, R. David, J. Ondrej, G. Drettakis, and P. Robert, "A Feasibility Study with ImageBased Rendered Virtual Reality in Patients with Mild Cognitive Impairment and Dementia," PLOS One, 18; 11(3), March 2016.

[31] M. Diehl, M. Marsiske, A.L. Horgas, A. Rosenberg, J. Saczynski, and S.L. Willis, "The Revised Observed Tasks of Daily Living: a performance-based assessment of everyday problem solving in older adults," J Appl Gerontol, 24:211-230, 2005.

[32] E. Quintana and J. Favela, "Augmented Reality Annotations to Assist Persons with Alzheimer's and Their Caregivers", Personal and Ubiquitous Computing," vol. 17, no. 6, pp. 1105-1116, 2012.

[33] Yao-Jen Chang, H. Liu, Y. Kang, C. Chian, and Yao-Sheng Chang, "Using augmented reality smart glasses to design games for cognitive training," $13^{\text {th }}$ International Conference on Remote Engineering and Virtual Instrumentation (REV), 252, 2016.

[34] T. Chandrasekera, M. Kang, P. Hebert, and P. Choo, "Augmenting space: enhancing health, safety, and well-being of older adults through hybrid spaces," Technology and Disability 29(3):141-151, 2017.

[35] A. Mihailidis , J. N. Boger, T. Craig, and J. Hoey, "The COACH prompting system to assist older adults with dementia through handwashing: An efficacy study," BMC Geriatr, 8:28, November 2008.

[36] S. Wood and R. J. McCrindle, "Augmented reality discovery and information system for people with memory loss," Personal and Ubiquitous Computing, 17(6), August 2013.

[37] P.C. Roy, A. Bouzouane, S. Giroux , and B. Bouchard, "Possibilistic activity recognition in smart homes for cognitively impaired people," Appl Artif Intell 25, 883-926, 2011.

[38] P. Dawadi, D. J. Cook, M. Schmitter-Edgecombe, and C. Parsey, "Automated assessment of cognitive health using smart home technologies," Technol Health Care, vol. 21, no. 4, pp. 323-343, 2013.

[39] K. Jekel, M. Damian, H. Storf, L. Hausner, and L. Frolich, "Development of a proxy-free objective assessment tool of instrumental activities of daily living in mild cognitive impairment using smart home technologies," J. Alzheimer's Dis., vol. 52, no. 2, pp. 509-517, May 2016.

[40] J. A. Kaye, S. A. Maxwell, N. Mattek, T. L. Hayes, H. Dodge, M. Pavel, H. B. Jimison, K. Wild, L. Boise, and T. A. Zitzelberger, "Intelligent systems for assessing aging changes: Home-based unobtrusive and continuous assessment of aging", J. Geron. B Psych. Sci., vol. 66B, pp. 180-190, July 2011.

[41] C. Boletsis and S. McCallum, "Augmented Reality Cubes for Cognitive Gaming: Preliminary Usability and Game Experience Testing,” Int. J. Serious Game, 3(1),3-18, 2016.

[42] L. Bonanni, C. H. Lee, and T. Selker, "Counter Intelligence: Augmented Reality Kitchen,” Proc. CHI, vol. 2239, p.44, 2005.

[43] https://www.nia.nih.gov/health/home-safety-and-alzheimers-disease

[44] C. Miranda-Castillo, B. Woods, and M. Orrell, "People with dementia living alone: what are their needs and what kind of support are they receiving?," Int Psychogeriatr, 22(4):607-17, June 2010. 